\title{
Approximately Optimal Utility Maximization
}

\author{
Angelia Nedić \\ IESE Department \\ University of Illinois \\ Urbana, IL 61801, USA. \\ Email: angelia@illinois.edu
}

\author{
Vijay G. Subramanian \\ Hamilton Institute \\ National University of Ireland, Maynooth \\ Co. Kildare, Ireland. \\ Email: vijay.subramanian@nuim.ie
}

\begin{abstract}
All opportunistic scheduling algorithms solve simpler optimization problems at each scheduling instance in order to achieve good long-term performance. The analysis of these algorithms assumes that the simpler optimization problems are solved exactly. However, in contrast, real-life implementations only approximately solve these problems but still yield close to optimal performance. We formalize this observation by explicitly bounding the longterm performance in terms of the error in the approximation made at every stage.
\end{abstract}

\section{INTRODUCTION}

The emergence of wireless data spurred on by developments in WLANs (802.11) and WANs (2.5/3/4G cellular technologies) has produced significant research on opportunistic scheduling in stochastic processing networks. An abbreviated list of the body of work in this area can be found in the following articles [20], [1], [12], [13], [3], [19], [18], [17], [6], [9], [7], where the scheduling decision can be mathematically abstracted as finding the control decision that maximizes the projection of the resulting rates vectors with the gradient of an appropriate utility function. In all of these it is demonstrated that exactly solving this optimization problem yields solutions that (asymptotically) achieve utility maximizing solutions, ensure stability of the underlying queueing processes or both. However, owing to tight timing requirements or computational complexity constraints, in real practice [2], [19], [10], [11] a close to optimal solution is what is used. Since the control decisions influence the evolution of the system, it is a priori not immediate that implementing a close to optimal solution to the resulting optimization problem at every decision instance will closely approximate the optimal solution. Restricting attention to throughput-based utility maximizing solutions, it has been shown [2], [19], [10], [11] for some examples and through simulations that one achieves a close to optimal solution by approximately solving the optimization problem at every decision instance. Our aim in this paper is to formally justify such a result for the throughput-based utility optimization problem in the general setting. Before proceeding we should point out to the reader that it was shown (using different methods) in [9, Sect. 4.7 \& Sect. 5.2 (Cor. 5.2)] that for a specific class of policies, sub-optimality of rate allocation within an additive constant yields performance within the additive constant while aiming for sub-optimality within a multiplicative constant $(x<1)$ can only provably guarantee performance relative to a scaled (by $x$ ) rate-region.

\section{The ModeL}

Consider a wireless communication system with $d$ users. The channel conditions are time varying and captured by a stochastic channel state $\eta_{k} \in \mathcal{S}$ at time $k$, where $\mathcal{S}$ is the channel state space; we assume that the channel state space $\mathcal{S}$ is finite, i.e., $\mathcal{S}=\{1, \ldots, m\}$ for some positive integer $m$. Associated with each state $\eta \in \mathcal{S}$ we have a rate-region $\mathcal{R}(\eta) \subset \mathbb{R}_{+}^{d}$. Thus when the channel is in the state $\eta$, the users may transmit at any vector of rates $\mathbf{r}=\left(r_{1}, \ldots, r_{d}\right) \in \mathcal{R}(\eta)$. We will assume throughout this paper that $\mathcal{R}(\eta) \subseteq K$ for some compact set $K \subset \mathbb{R}_{+}^{d}$ and for all $\eta \in \mathcal{S}$. We will also assume that the process $\left\{\eta_{k}\right\}$ evolves according to an ergodic homogeneous Markov chain with transition probability matrix $P$, i.e., for all $k \geq 0$,

$\operatorname{Prob}\left\{\eta_{k+1}=j \mid \eta_{k}=i\right\}=P_{i j}, \quad \forall i, j \in\{1, \ldots, m\}$, and with the stationary distribution vector $\gamma \in \mathbb{R}_{++}^{m}$. We will further assume that $\mathcal{R}(\eta)$ is convex and closed for every $\eta$. Then, it can be seen that the steady-state rate region $\overline{\mathcal{R}}$, given by

$$
\overline{\mathcal{R}}=\left\{\sum_{j=1}^{m} \gamma_{j} w_{j} \mid w_{j} \in \mathcal{R}(j), \forall j=1, \ldots, m\right\},
$$

is also convex and compact. Note that $\overline{\mathcal{R}}$ is precisely the set of all achievable steady-state long-term empirical throughput vectors. See [2], [19], [10], [11] for examples of some specific wireless communication systems including TDMA and CDMA cellular systems and adhoc networks that fit the above mathematical model.

We assume that the $d$ users are rate-adaptive and need to share the channel described above, fairly and efficiently. The problem that we would like to solve can be translated into the following utility maximization:

$$
\sup _{\mathbf{w} \in \overline{\mathcal{R}}} \sum_{i=1}^{d} U_{i}\left(w_{i}\right)(\triangleq U(\mathbf{w}))
$$


where each function $U_{i}(\cdot)$ is concave and continuously differentiable on $\Re_{+}$. From the above observations on $\overline{\mathcal{R}}$, it follows that a maximizer exists.

\section{A Gradient Based Scheduling Algorithm}

Let $\mathbf{R}_{k}=\left(R_{1, k}, \ldots, R_{d, k}\right)^{T} \in \mathcal{R}\left(\eta_{k}\right)$ be the rate selected at time $k$. Define $\mathbf{W}_{k}=\left(W_{1, k}, \ldots, W_{d, k}\right)^{T}$ to be the empirical throughput as follows:

$$
\mathbf{W}_{k+1}=\mathbf{W}_{k}+\mu_{k}\left(\mathbf{R}_{k}-\mathbf{W}_{k}\right), \quad k \geq 0,
$$

starting with $\mathbf{W}_{0}=0$. In this paper, we consider a diminishing step-size viz., $\mu_{k}=1 /(k+1), k \geq 0$, and we are interested in the value of $U\left(\mathbf{W}_{k}\right)$ as $k \rightarrow+\infty$.

To do so, one usually considers a myopic view of the optimization problem: optimize $U\left(\mathbf{W}_{k+1}\right)$ by choosing $\mathbf{R}_{k} \in \mathcal{R}\left(\eta_{k}\right)$ appropriately given that $\mathbf{R}_{0}, \ldots, \mathbf{R}_{k-1}$ have already been chosen. Note that for $\mu_{k} \ll 1$,

$$
U\left(\mathbf{W}_{k+1}\right)-U\left(\mathbf{W}_{k}\right) \approx \mu_{k} \nabla U\left(\mathbf{W}_{k}\right)^{T}\left(\mathbf{R}_{k}-\mathbf{W}_{k}\right),
$$

Thus, for small enough $\mu_{k}$ the best choice given the past decisions is to choose a point $\mathbf{R}_{k}$ in the capacity region that satisfies $\mathbf{R}_{k}=\arg \max _{\mathbf{r} \in \mathcal{R}\left(\eta_{k}\right)} \nabla U\left(\mathbf{W}_{k}\right)^{T} \mathbf{r}$. This defines a set-valued function $H_{\eta}(\mathbf{w})$, given by

$$
H_{\eta}(\mathbf{w})=\arg \max _{\mathbf{r} \in \mathcal{R}(\eta)} \nabla U(\mathbf{w})^{T} \mathbf{r},
$$

for $\mathbf{w} \in \mathbb{R}_{+}^{d}$ and for every channel state $\eta \in \mathcal{S}$.

Remark We make the following observations:

1) This leads to a gradient-based scheduling algorithm. In the optimization literature this is the conditional gradient or Frank-Wolfe algorithm [4].

2) With the convex rate region assumption, this is a convex problem at each scheduling instance.

3) When the region is a simplex for every state, we obtain a TDM-type algorithm where only one user is allowed to transmit at a time.

Often owing to tight timing requirements or computational complexity restrictions, we do not solve for an optimal solution to (3) but select a solution from the $\epsilon_{k^{-}}$ optimal set $\tilde{H}_{\eta}(\mathbf{w}) \subseteq \mathcal{R}(\eta)$ such that for all $\mathbf{v} \in \tilde{H}(\mathbf{w})$,

$$
\nabla U(\mathbf{w})^{T} \mathbf{v} \geq \max _{\mathbf{r} \in \mathcal{R}\left(\eta_{k}\right)} \nabla U(\mathbf{w})^{T} \mathbf{r}-\epsilon_{k} .
$$

We let $\tilde{\mathbf{W}}_{k}$ be the empirical throughput up to time $k$ and set $\tilde{\mathbf{V}}_{k} \in \tilde{H}_{\eta_{k}}\left(\tilde{\mathbf{W}}_{k}\right)$ to be the rate selected at time $k$. Then, we update the empirical throughputs as follows:

$$
\tilde{\mathbf{W}}_{k+1}=\tilde{\mathbf{W}}_{k}+\mu_{k}\left(\tilde{\mathbf{V}}_{k}-\tilde{\mathbf{W}}_{k}\right), \quad k \geq 0,
$$

with $\tilde{\mathbf{W}}_{0}=0$. We would like to understand the behavior of $U\left(\tilde{\mathbf{W}}_{k}\right)$ as $k \rightarrow+\infty$. In particular, we would like to identify the limit points of $U\left(\tilde{\mathbf{W}}_{k}\right)$ when $\epsilon_{k} \rightarrow \epsilon$.

\section{Analysis of Suboptimal Algorithm}

In our analysis of the algorithm (5), we use some well-known results as well as some implications of these results, which are given in the following subsection.

\section{A. Preliminaries}

We give some convergence results on Markov chains, a set sequence and a scalar sequence.

1) Results on Markov chains: For an ergodic homogeneous Markov chain with transition matrix $P$, the matrix $P^{k}$ converges, as $k \rightarrow \infty$, to a matrix whose each row is equal to the steady state distribution $\gamma \in \mathbb{R}_{++}^{m}$. The results that we will use are: (a) For all $i \in\{1, \ldots, m\}$, the entries $P_{i j}^{k}$ converge to $\gamma_{j}$ with a geometric rate, i.e.,

$$
\left|P_{i j}^{k}-\gamma_{j}\right| \leq M_{0} \rho^{k}, \quad \forall j=1, \ldots, m, \forall k \geq 0,
$$

where $M_{0}$ and $\rho$ are some scalars with $M_{0}>0$ and $\rho \in(0,1)$; and (b) if $\kappa_{k}(j)$ is the number of visits to state $j$ up to time $k$, then for any initial distribution on states, with probability 1 ,

$$
\lim _{k \rightarrow \infty} \frac{\kappa_{k}(j)}{k+1}=\gamma_{j}, \quad \forall j=1, \ldots, m .
$$

The result in part (a) can be found in [14] (see there Section 6-10), while the result in part (b) can be found in [8] (see there Theorem 1 on page 154).

2) Set convergence: Given a sequence of nonempty sets $\left\{C_{k}\right\}$ with $C_{k} \subseteq \mathbb{R}^{d}$ for all $k$, the outer limit and the inner limit of the set sequence $\left\{C_{k}\right\}$ are the sets $\limsup _{k \rightarrow \infty} C_{k}$ and $\liminf _{k \rightarrow \infty} C_{k}$, which are defined as follows (see [16], Chapter 4):

$$
\begin{aligned}
\limsup _{k \rightarrow \infty} C_{k}=\left\{x \mid \exists\left\{x_{k}\right\}_{\mathcal{K}} \text { s.t. } \lim _{\substack{k \rightarrow \infty \\
k \in \mathcal{K}}} x_{k}=x\right. \\
\left.\quad \text { and } x_{k} \in C_{k}, \forall k \in \mathcal{K}\right\}, \\
\liminf _{k \rightarrow \infty} C_{k}=\left\{x \mid \exists\left\{x_{k}\right\} \text { s.t. } \lim _{k \rightarrow \infty} x_{k}=x\right. \\
\text { and } \left.x_{k} \in C_{k}, \forall k \geq \bar{k} \text { for some } \bar{k}\right\} .
\end{aligned}
$$

When the outer and the inner limit are equal, we say that the set sequence $C_{k}$ is convergent. We denote the limit set by $C$ and we write $\lim _{k \rightarrow \infty} C_{k}=C$.

We next discuss a convergence property of a weighted sum of closed sets, in fact compact sets. For each $j=1, \ldots, M$, we assume that $\left\{u_{j, k}\right\}$ is a random scalar sequence such that $\lim _{k \rightarrow \infty} u_{j, k}=\hat{u}_{j}$ with probability 1. The set convergence result that we will use (without proof) is the following: if $\left\{X_{j}, j=1, \ldots, M\right\}$ is a finite collection of nonempty closed sets in $\mathbb{R}^{d}$ that are contained in a compact superset $K \subset \mathbb{R}^{d}$, then the sequence of sets $\left\{\sum_{j=1}^{M} u_{j, k} X_{j}\right\}$ converges to the set $\sum_{j=1}^{m} \hat{u}_{j} X_{j}$ with probability 1 , i.e.,

$$
\lim _{k \rightarrow \infty} \sum_{j=1}^{M} u_{j, k} X_{j}=\sum_{j=1}^{m} \hat{u}_{j} X_{j} \quad \text { w.p. } 1 .
$$

3) Scalar sequence convergence: The following result on convergence of a scalar sequence can be found for example in [15], Chapter 2.

Lemma 1. Let $\left\{u_{k}\right\}$ be a scalar sequence satisfying the following relation

$$
u_{k+1} \leq q_{k} u_{k}+\alpha_{k} \quad \forall k \geq 0,
$$


where $q_{k} \in[0,1)$ and $\alpha_{k} \geq 0$ for all $k, \sum_{k=0}^{\infty}\left(1-q_{k}\right)=$ $\infty$, and $\lim _{k \rightarrow \infty} \frac{\alpha_{k}}{1-q_{k}}=0$. Then, $\lim \sup _{k \rightarrow \infty} u_{k} \leq 0$.

\section{B. Convergence Analysis}

For ease of exposition and to more easily refer to the optimization literature, we will work with $f(\cdot)=-U(\cdot)$ Thus, method (4)-(5) reduces to:

$$
\tilde{\mathbf{W}}_{k+1}=\tilde{\mathbf{W}}_{k}+\mu_{k}\left(\tilde{\mathbf{V}}_{k}-\tilde{\mathbf{W}}_{k}\right), \quad k \geq 0,
$$

with $\tilde{\mathbf{W}}_{0}=0$, and $\tilde{\mathbf{V}}_{k} \in \mathcal{R}\left(\eta_{k}\right)$ such that

$$
\nabla f\left(\tilde{\mathbf{W}}_{k}\right)^{T} \tilde{\mathbf{V}}_{k} \leq \min _{\mathbf{r} \in \mathcal{R}\left(\eta_{k}\right)} \nabla f\left(\tilde{\mathbf{W}}_{k}\right)^{T} \mathbf{r}+\epsilon_{k},
$$

where $\epsilon_{k}>0$.

We consider the properties of the sequences $\left\{\tilde{\mathbf{V}}_{k}\right\}$ and $\left\{\tilde{\mathbf{W}}_{k}\right\}$ under the following assumptions.

Assumption 1. The sets $R(j) \subset \mathbb{R}^{d}, j=1, \ldots, m$, are convex, closed and contain the origin. Moreover, each set $\mathcal{R}(j)$ is contained in a compact superset $K \subset \mathbb{R}^{d}$.

Assumption 2. The function $f$ is convex and continuously differentiable on $\mathbb{R}_{+}^{d}$. Furthermore, its gradient $\nabla f(x)$ is Lipschitz continuous with constant L, i.e.,

$$
\|\nabla f(x)-\nabla f(y)\| \leq L\|x-y\|, \quad \forall x, y \in \mathbb{R}_{+}^{d} .
$$

By Assumption 1 the average rate region $\overline{\mathcal{R}}$, as given in (1), is convex and compact. Thus, an optimal solution for the problem $\min _{w \in \overline{\mathcal{R}}} f(w)$ exists, but need not be unique. It is unique when $f$ is strictly or strongly convex.

When $f$ is differentiable with Lipschitz gradients, we have the following relation for all $x, y \in \mathbb{R}^{d}$,

$$
f(y) \leq f(x)+\nabla f(x)^{T}(y-x)+\frac{L}{2}\|x-y\|^{2},
$$

where $L>0$ is the Lipschitz constant for the gradients (see e.g., [4, Prop. A.24, pg. 667]).

Using descent relation (11), we establish a basic relation for the values $f\left(\tilde{\mathbf{W}}_{k+1}\right)$, as given in the following.

Lemma 2. Let Assumptions 1 and 2 hold. Let $\mu_{k} \in[0,1]$ for all $k$ in Eq. (9), and let $\left\{\epsilon_{k}\right\}$ be any positive scalar sequence. Then, we surely have:

(a) The sequences $\left\{\tilde{\mathbf{V}}_{k}\right\}$ and $\left\{\tilde{\mathbf{W}}_{k}\right\}$ are bounded. In particular, $\left\{\tilde{\mathbf{W}}_{k}\right\}$ is contained in the compact set $\operatorname{conv}(K)$.

(b) For all $k \geq 0$,

$$
\begin{aligned}
f\left(\tilde{\mathbf{W}}_{k+1}\right) \leq & f\left(\tilde{\mathbf{W}}_{k}\right)+2 \mu_{k}^{2} L D^{2} \\
& +\mu_{k} \nabla f\left(\tilde{\mathbf{W}}_{k}\right)^{T}\left(\tilde{\mathbf{V}}_{k}-\tilde{\mathbf{W}}_{k}\right),
\end{aligned}
$$

where $D:=\max _{u \in \operatorname{conv}(K)}\|u\|$.

Proof: Since $\tilde{\mathbf{V}}_{k} \in \mathcal{R}\left(\eta_{k}\right) \subset K$ for all $k$, it follows that $\left\{\tilde{\mathbf{V}}_{k}\right\} \subset K$. From the definition of $\tilde{\mathbf{W}}_{k}$ in 9 , we have for all $k \geq 0$,

$$
\begin{gathered}
\tilde{\mathbf{W}}_{k+1}=\mu_{k} \tilde{\mathbf{V}}_{k}+\left(\sum_{t=0}^{k-1}\left(1-\mu_{k}\right) \cdots\left(1-\mu_{t+1}\right) \mu_{t} \tilde{\mathbf{V}}_{t}\right) \\
+\left(1-\mu_{k}\right) \cdots\left(1-\mu_{0}\right) \tilde{\mathbf{W}}_{0}
\end{gathered}
$$

When $\mu_{k} \in[0,1]$ for all $k$, each $\tilde{\mathbf{W}}_{k}$ is a convex combination of $\tilde{\mathbf{W}}_{0}$ and the vectors $\tilde{\mathbf{V}}_{\ell}$ for $\ell=0, \ldots, k-1$, all of which belong to the set $K$. It follows that each $\tilde{\mathbf{W}}_{k}$ belongs to the convex hull of $K$, i.e., $\tilde{\mathbf{W}}_{k} \in \operatorname{conv}(K)$ for all $k$. Since $K$ is compact, so is its convex hull (see [5], Proposition 1.3.2).

Using descent relation (11), we obtain for all $k$,

$$
\begin{aligned}
f\left(\tilde{\mathbf{W}}_{k+1}\right) \leq & f\left(\tilde{\mathbf{W}}_{k}\right)+\frac{L}{2}\left\|\tilde{\mathbf{W}}_{k+1}-\tilde{\mathbf{W}}_{k}\right\|^{2} \\
& +\nabla f\left(\tilde{\mathbf{W}}_{k}\right)^{T}\left(\tilde{\mathbf{W}}_{k+1}-\tilde{\mathbf{W}}_{k}\right) .
\end{aligned}
$$

Since $\tilde{\mathbf{W}}_{k+1}-\tilde{\mathbf{W}}_{k}=\mu_{k}\left(\tilde{\mathbf{V}}_{k}-\tilde{\mathbf{W}}_{k}\right)$ (see relation (9)), we have for all $k$,

$$
\begin{aligned}
f\left(\tilde{\mathbf{W}}_{k+1}\right) \leq & f\left(\tilde{\mathbf{W}}_{k}\right)+\mu_{k}^{2} \frac{L}{2}\left\|\tilde{\mathbf{V}}_{k}-\tilde{\mathbf{W}}_{k}\right\|^{2} \\
& +\mu_{k} \nabla f\left(\tilde{\mathbf{W}}_{k}\right)^{T}\left(\tilde{\mathbf{V}}_{k}-\tilde{\mathbf{W}}_{k}\right) .
\end{aligned}
$$

In view of $V_{k} \in K$ and $\tilde{\mathbf{W}}_{k} \in \operatorname{conv}(K)$ for all $k$, it follows that $\left\|\tilde{\mathbf{V}}_{k}-\tilde{\mathbf{W}}_{k}\right\| \leq 2 D$ for all $k$, with $D=$ $\max _{u \in \operatorname{conv}(K)}\|u\|$, implying the desired relation.

\section{Diminishing Step-size}

Consider algorithm (9)-(10) with the step-size given by $\mu_{k}=\frac{1}{k+1}$ for all $k$. From the definition of $\tilde{\mathbf{W}}_{k}$ in (9), it can be seen that for all $k \geq 1$ and $0 \leq t<k$,

$$
\tilde{\mathbf{W}}_{k+1}=\frac{t}{k+1} \tilde{\mathbf{W}}_{t}+\frac{1}{k+1} \sum_{s=t}^{k} \tilde{\mathbf{V}}_{s} .
$$

For this step-size, we show that the accumulation points of the throughput sequence $\left\{\tilde{\mathbf{W}}_{k}\right\}$ belong to the steady-state rate region $\overline{\mathcal{R}}$, as seen in the following.

Lemma 3. Let Assumption 1 hold. Then, with probability 1 , all of the accumulation points of $\left\{\tilde{\mathbf{W}}_{k}\right\}$ belong to the steady-state rate region $\overline{\mathcal{R}}$.

Proof: Using relation (12) we can show that $\tilde{\mathbf{W}}_{k+1} \in \sum_{j=1}^{m} \frac{\kappa_{k}(j)}{k+1} \mathcal{R}(j)$. The result then follows by using relations (7) and (8).

We now show that all accumulation points of the sequence are $\epsilon$-optimal, provided that the errors $\epsilon_{k} \rightarrow \epsilon$.

Theorem 1. Let Assumptions 1 and 2 hold. Let $\left\{\tilde{\mathbf{W}}_{k}\right\}$ be the iterate sequence generated by method (9)-(10) with $\mu_{k}=\frac{1}{k+1}$ and the nonnegative scalar sequence $\left\{\epsilon_{k}\right\}$ such that $\lim _{k \rightarrow \infty} \epsilon_{k}=\epsilon$ and $\lim _{k \rightarrow \infty} \frac{\epsilon_{k}-\epsilon_{k+1}}{\mu_{k}}=0$. Then,

$$
\limsup _{k \rightarrow \infty} E\left[f\left(\tilde{\mathbf{W}}_{k}\right)\right] \leq f^{*}+\epsilon .
$$

Proof: Since $f$ is continuous over the compact set $\overline{\mathcal{R}}$, there exists $\mathbf{x}^{*} \in \overline{\mathcal{R}}$ attaining the minimum of $f$ over $\overline{\mathcal{R}}$. Moreover, since $\overline{\mathcal{R}}=\sum_{j=1}^{m} \gamma_{j} \mathcal{R}(j)$, there exist vectors $x_{j}^{*} \in \mathcal{R}(j), j=1, \ldots, m$ such that

$$
\mathbf{x}^{*}=\sum_{j=1}^{m} \gamma_{j} \mathbf{x}_{j}^{*}
$$


Define the random sequence $\left\{\mathbf{r}_{k}^{*}\right\}$ as follows:

$$
\mathbf{r}_{k}^{*}=\mathbf{x}_{j}^{*} \text { when } \eta_{k}=j, \quad \forall k \geq 0 .
$$

By the definition of $\tilde{\mathbf{V}}_{k}$ in 10 and the relation

$\min _{\mathbf{r} \in \mathcal{R}\left(\eta_{k}\right)} \nabla f\left(\tilde{\mathbf{W}}_{k}\right)^{T}\left(\mathbf{r}-\tilde{\mathbf{W}}_{k}\right) \leq \nabla f\left(\tilde{\mathbf{W}}_{k}\right)^{T}\left(\mathbf{r}_{k}^{*}-\tilde{\mathbf{W}}_{k}\right)$,

we obtain for all $k$,

$\nabla f\left(\tilde{\mathbf{W}}_{k}\right)^{T}\left(\tilde{\mathbf{V}}_{k}-\tilde{\mathbf{W}}_{k}\right) \leq \nabla f\left(\tilde{\mathbf{W}}_{k}\right)^{T}\left(\mathbf{r}_{k}^{*}-\tilde{\mathbf{W}}_{k}\right)+\epsilon_{k}$.

By combining the preceding relation with the inequality of Lemma 2, we surely have for all $k \geq 0$,

$$
\begin{aligned}
f\left(\tilde{\mathbf{W}}_{k+1}\right) \leq & f\left(\tilde{\mathbf{W}}_{k}\right)+\mu_{k} \epsilon_{k}+2 \mu_{k}^{2} L D^{2} \\
& +\mu_{k} \nabla f\left(\tilde{\mathbf{W}}_{k}\right)^{T}\left(\mathbf{r}_{k}^{*}-\tilde{\mathbf{W}}_{k}\right),
\end{aligned}
$$

where $D=\max _{u \in \operatorname{conv}(K)}\|u\|$.

We now estimate $\nabla f\left(\tilde{\mathbf{W}}_{k}\right)^{T}\left(\mathbf{r}_{k}^{*}-\tilde{\mathbf{W}}_{k}\right)$ using the past throughput $\tilde{\mathbf{W}}_{t}$ for some $t<k$, enabling us to exploit the properties of the underlying Markov chain. For any $t<k$, we have

$$
\begin{aligned}
\nabla & f\left(\tilde{\mathbf{W}}_{k}\right)^{T}\left(\mathbf{r}_{k}^{*}-\tilde{\mathbf{W}}_{k}\right) \\
\leq & \left|\left(\nabla f\left(\tilde{\mathbf{W}}_{k}\right)-\nabla f\left(\tilde{\mathbf{W}}_{t}\right)\right)^{T}\left(\mathbf{r}_{k}^{*}-\tilde{\mathbf{W}}_{k}\right)\right| \\
& +\nabla f\left(\tilde{\mathbf{W}}_{t}\right)^{T}\left(\mathbf{r}_{k}^{*}-\tilde{\mathbf{W}}_{k}\right) .
\end{aligned}
$$

Using the Cauchy-Schwartz inequality and the Lipschitz gradient property of $f$, we further have

$\left|\left(\nabla f\left(\tilde{\mathbf{W}}_{k}\right)-\nabla f\left(\tilde{\mathbf{W}}_{t}\right)\right)^{T}\left(\mathbf{r}_{k}^{*}-\tilde{\mathbf{W}}_{k}\right)\right| \leq 2 L D\left\|\tilde{\mathbf{W}}_{k}-\tilde{\mathbf{W}}_{t}\right\|$,

where in the last inequality we use $\tilde{\mathbf{W}}_{k} \in \operatorname{conv}(K)$ and $r_{k}^{*} \in K$ for all $k$, and $D$ denotes the maximum norm of the vectors in the set $\operatorname{conv}(K)$. Thus, we have

$$
\begin{aligned}
& \nabla f\left(\tilde{\mathbf{W}}_{k}\right)^{T}\left(\mathbf{r}_{k}^{*}-\tilde{\mathbf{W}}_{k}\right) \leq \nabla f\left(\tilde{\mathbf{W}}_{t}\right)^{T}\left(\mathbf{r}_{k}^{*}-\tilde{\mathbf{W}}_{t}\right) \\
& \quad+\nabla f\left(\tilde{\mathbf{W}}_{t}\right)^{T}\left(\tilde{\mathbf{W}}_{t}-\tilde{\mathbf{W}}_{k}\right)+2 L D\left\|\tilde{\mathbf{W}}_{k}-\tilde{\mathbf{W}}(t)\right\| .
\end{aligned}
$$

For the term $\nabla f\left(\tilde{\mathbf{W}}_{t}\right)^{T}\left(\tilde{\mathbf{W}}_{t}-\tilde{\mathbf{W}}_{k}\right)$ we have

$$
\nabla f\left(\tilde{\mathbf{W}}_{t}\right)^{T}\left(\tilde{\mathbf{W}}_{t}-\tilde{\mathbf{W}}_{k}\right) \leq C\left\|\tilde{\mathbf{W}}_{t}-\tilde{\mathbf{W}}_{k}\right\|,
$$

where $C=\max _{x \in \operatorname{conv}(K)}\|\nabla f(x)\|$, which is finite by the continuity of $\nabla f$ and compactness of $\operatorname{conv}(K)$. Hence, for all $k>0$ and $0 \leq t \leq k$,

$$
\begin{aligned}
& \nabla f\left(\tilde{\mathbf{W}}_{k}\right)^{T}\left(\mathbf{r}_{k}^{*}-\tilde{\mathbf{W}}_{k}\right) \leq \\
& c_{1}\left\|\tilde{\mathbf{W}}_{k}-\tilde{\mathbf{W}}_{t}\right\|+\nabla f\left(\tilde{\mathbf{W}}_{t}\right)^{T}\left(\mathbf{r}_{k}^{*}-\tilde{\mathbf{W}}_{t}\right),
\end{aligned}
$$

where $c_{1}=2 L D+C$.

Now, we estimate $\left\|\tilde{\mathbf{W}}_{k}-\tilde{\mathbf{W}}_{t}\right\|$. From relation 12, we have for $k \geq 1$ and $0 \leq t<k$,

$$
\tilde{\mathbf{W}}_{k+1}-\tilde{\mathbf{W}}_{t}=\frac{t-k-1}{k+1} \tilde{\mathbf{W}}_{t}+\frac{1}{k+1} \sum_{s=t}^{k} \tilde{\mathbf{V}}_{s}
$$

The vector $\frac{k+1-t}{k+1} \frac{1}{k+1-t} \sum_{s=t}^{k} \tilde{\mathbf{V}}_{s}$ is a scaled convex combination of vectors $\tilde{\mathbf{V}}_{s} \in K, s=t, \ldots, k$, hence it belongs to $\frac{k+1-t}{k+1} \operatorname{conv}(K)$. Since $\tilde{\mathbf{W}}_{k} \in \operatorname{conv}(K)$, we have $\left\|\frac{1}{k+1-t} \sum_{s=t}^{k} \tilde{\mathbf{V}}_{s}-\tilde{\mathbf{W}}_{t}\right\| \leq 2 D$, implying that for all $k \geq 1$ and $0 \leq t<k$,

$$
\left\|\tilde{\mathbf{W}}_{k+1}-\tilde{\mathbf{W}}_{t}\right\| \leq \frac{k+1-t}{k+1} 2 D .
$$

Substituting the preceding estimate in (16), we obtain

$$
\begin{aligned}
\nabla f\left(\tilde{\mathbf{W}}_{k}\right)^{T}\left(\mathbf{r}_{k}^{*}-\tilde{\mathbf{W}}_{k}\right) \leq & \frac{k+1-t}{k+1} c_{2}+ \\
& \nabla f\left(\tilde{\mathbf{W}}_{t}\right)^{T}\left(\mathbf{r}_{k}^{*}-\tilde{\mathbf{W}}_{t}\right),
\end{aligned}
$$

where $c_{2}=2 D c_{1}$. Finally, by combining the preceding inequality with relation (15), we obtain for all $k \geq 1$ and any $t$ with $0 \leq t<k$,

$$
\begin{aligned}
& f\left(\tilde{\mathbf{W}}_{k+1}\right) \leq f\left(\tilde{\mathbf{W}}_{k}\right)+\mu_{k} \epsilon_{k}+2 \mu_{k}^{2} L D^{2} \\
& \quad+\mu_{k} \nabla f\left(\tilde{\mathbf{W}}_{t}\right)^{T}\left(\mathbf{r}_{k}^{*}-\tilde{\mathbf{W}}_{t}\right)+\mu_{k} \frac{k+1-t}{k+1} c_{2} .
\end{aligned}
$$

Let $\mathcal{F}_{k}$ denote the $\sigma$-field generated by $\left\{\eta_{s}\right\}_{s=0}^{k}$. Taking the expectation conditioned on $\mathcal{F}_{t}$, with $0 \leq t<k$, from relation (18) we have

$$
\begin{aligned}
E\left[f\left(\tilde{\mathbf{W}}_{k+1}\right) \mid \mathcal{F}_{t}\right] \leq & E\left[f\left(\tilde{\mathbf{W}}_{k}\right) \mid \mathcal{F}_{t}\right]+2 \mu_{k}^{2} L D^{2} \\
& +\mu_{k} \nabla f\left(\tilde{\mathbf{W}}_{t}\right)^{T}\left(E\left[\mathbf{r}_{k}^{*} \mid \mathcal{F}_{t}\right]-\tilde{\mathbf{W}}_{t}\right) \\
& +\mu_{k} \frac{k+1-t}{k+1} c_{2}+\mu_{k} \epsilon_{k} .
\end{aligned}
$$

We now estimate the term $\nabla f\left(\tilde{\mathbf{W}}_{t}\right)^{T}\left(E\left[\mathbf{r}_{k}^{*} \mid \mathcal{F}_{t}\right]-\right.$ $\left.\tilde{\mathbf{W}}_{t}\right)$ for $t<k$. By the definition of process $\left\{r_{k}^{*}\right\}$ and the ergodicity of $\left\{\eta_{k}\right\}$, it follows that

$E\left[\mathbf{r}_{k}^{*} \mid \mathcal{F}_{t}\right]=\sum_{j=1}^{m} P_{\eta_{t}, j}^{k-t} x_{j}^{*}=\sum_{j=1}^{m} \gamma_{j} \mathbf{x}_{j}^{*}+\sum_{j=1}^{m}\left(P_{\eta_{t}, j}^{k-t}-\gamma_{j}\right) \mathbf{x}_{j}^{*}$.

Since $\sum_{j=1}^{m} \gamma_{j} \mathbf{x}_{j}^{*}=\mathbf{x}^{*}$ (see Eq. 13p), it follows

$$
\begin{gathered}
\nabla f\left(\tilde{\mathbf{W}}_{t}\right)^{T}\left(E\left[\mathbf{r}_{k}^{*} \mid \mathcal{F}_{t}\right]-\tilde{\mathbf{W}}_{t}\right) \leq \nabla f\left(\tilde{\mathbf{W}}_{t}\right)^{T}\left(\mathbf{x}^{*}-\tilde{\mathbf{W}}_{t}\right) \\
+\left\|\nabla f\left(\tilde{\mathbf{W}}_{t}\right)\right\|\left(\max _{1 \leq \ell \leq m}\left|P_{\eta_{t}, \ell}^{k-t}-\gamma_{\ell}\right|\right) \sum_{j=1}^{m}\left\|\mathbf{x}_{j}^{*}\right\| .
\end{gathered}
$$

By relation (6), we have $\left|P_{i \ell}^{k-t}-\gamma_{j}\right| \leq M_{0} \rho^{k-t}$ for any $i, \ell=1, \ldots, m$. Furthermore, by the compactness of $\operatorname{conv}(K)$ and the continuity of $\nabla f$, we have that $\max _{w \in \operatorname{conv}(K)}\|\nabla f(w)\|=C$ is finite. Also, since $\mathbf{x}_{j}^{*} \in R(j) \subset K$, it follows $\left\|\mathbf{x}_{j}^{*}\right\| \leq D$. Therefore,

$$
\begin{aligned}
& \nabla f\left(\tilde{\mathbf{W}}_{t}\right)^{T}\left(E\left[\mathbf{r}_{k}^{*} \mid \mathcal{F}_{t}\right]-\tilde{\mathbf{W}}_{t}\right) \leq \\
& \quad \nabla f\left(\tilde{\mathbf{W}}_{t}\right)^{T}\left(\mathbf{x}^{*}-\tilde{\mathbf{W}}_{t}\right)+c_{3} \rho^{k-t},
\end{aligned}
$$

with $c_{3}=C M_{0} m D$. Further, by convexity of $f$, we have

$$
\begin{aligned}
& \nabla f\left(\tilde{\mathbf{W}}_{t}\right)^{T}\left(\mathbf{x}^{*}-\tilde{\mathbf{W}}_{t}\right) \leq f\left(\mathbf{x}^{*}\right)-f\left(\tilde{\mathbf{W}}_{t}\right) \\
& \leq f\left(\mathbf{x}^{*}\right)-f\left(\tilde{\mathbf{W}}_{k}\right)+\left\|\nabla f\left(\tilde{\mathbf{W}}_{k}\right)\right\|\left\|\tilde{\mathbf{W}}_{k}-\tilde{\mathbf{W}}_{t}\right\| .
\end{aligned}
$$

The gradients $\nabla f$ are uniformly bounded over the set $\operatorname{conv}(K)$ by the scalar $C$. Therefore, by relation (17),

$$
\nabla f\left(\tilde{\mathbf{W}}_{t}\right)^{T}\left(\mathbf{x}^{*}-\tilde{\mathbf{W}}_{t}\right) \leq f\left(\mathbf{x}^{*}\right)-f\left(\tilde{\mathbf{W}}_{k}\right)+\frac{k-t}{k} c_{4},
$$


with $c_{4}=2 C D$. Thus, for all $k, t$ with $0 \leq t<k$,

$$
\begin{aligned}
& \nabla f\left(\tilde{\mathbf{W}}_{t}\right)^{T}\left(E\left[\mathbf{r}_{k}^{*} \mid \mathcal{F}_{t}\right]-\tilde{\mathbf{W}}_{t}\right) \leq \\
& \quad f\left(\mathbf{x}^{*}\right)-f\left(\tilde{\mathbf{W}}_{k}\right)+\frac{k-t}{k} c_{4}+c_{3} \rho^{k-t} .
\end{aligned}
$$

Using the preceding estimate in relation (19), we obtain

$$
\begin{aligned}
& E\left[f\left(\tilde{\mathbf{W}}_{k+1}\right) \mid \mathcal{F}_{t}\right] \leq E\left[f\left(\tilde{\mathbf{W}}_{k}\right) \mid \mathcal{F}_{t}\right] \\
& \quad+\mu_{k}\left(f\left(\mathbf{x}^{*}\right)-f\left(\tilde{\mathbf{W}}_{k}\right)\right)+\mu_{k} \frac{k+1-t}{k+1} c_{2} \\
& \quad+\mu_{k} \frac{k-t}{k} c_{4}+\mu_{k} c_{3} \rho^{k-t}+\mu_{k} \epsilon_{k}+2 \mu_{k}^{2} L D^{2} .
\end{aligned}
$$

By taking the total expectation, using $\frac{k+1-t}{k+1} \leq \frac{k-t}{k}+$ $\frac{1}{k+1}$, subtracting the term $f^{*}+\epsilon_{k+1}$ from both sides of the preceding relation, it can be seen that for all $k>0$ and any $t$ with $0 \leq t<k$,

$$
\begin{aligned}
& E\left[f\left(\tilde{\mathbf{W}}_{k+1}\right)\right]-f^{*}-\epsilon_{k+1} \\
& \leq\left(1-\mu_{k}\right)\left(E\left[f\left(\tilde{\mathbf{W}}_{k}\right)\right]-f^{*}-\epsilon_{k}\right)+\left(\epsilon_{k}-\epsilon_{k+1}\right) \\
& \quad+\mu_{k} c\left(\frac{k-t}{k}+\frac{1}{k+1}+\rho^{k-t}+\mu_{k}\right)
\end{aligned}
$$

where $c=\max \left\{c_{2}+c_{4}, c_{3}, 2 L D^{2}\right\}$. For $t=k-\lceil k\rceil^{\beta}$ with $\beta \in(0,1)$, relation 20 reduces to

$$
\begin{aligned}
E\left[f\left(\tilde{\mathbf{W}}_{k+1}\right)\right] & -f^{*}-\epsilon_{k+1} \leq\left(\epsilon_{k}-\epsilon_{k+1}\right)+\mu_{k} c a_{k} \\
& +\left(1-\mu_{k}\right)\left(E\left[f\left(\tilde{\mathbf{W}}_{k}\right)\right]-f^{*}-\epsilon_{k}\right),
\end{aligned}
$$

with $a_{k}=\frac{\lceil k\rceil^{\beta}}{k}+\frac{1}{k+1}+\rho^{\lceil k\rceil^{\beta}}+\mu_{k}$.

Using $\sum_{k} \mu_{k}=\infty, \lim _{k \rightarrow \infty} \frac{\epsilon_{k}-\epsilon_{k+1}}{\mu_{k}}=0$, and $\lim _{k \rightarrow \infty} a_{k}=0$, we can see that the conditions of Lemma 1 are satisfied, with the following identification: $u_{k}=E\left[f\left(\tilde{\mathbf{W}}_{k}\right)\right]-f^{*}-\epsilon_{k}, q_{k}=1-\mu_{k}$, and $\alpha_{k}=\left(\epsilon_{k}-\epsilon_{k+1}\right)+\mu_{k} c a_{k}$. By Lemma 1, it follows that $\limsup _{k \rightarrow \infty}\left(E\left[f\left(\tilde{\mathbf{W}}_{k}\right)\right]-f^{*}-\epsilon_{k}\right) \leq 0$, which in turn yields

$$
\limsup _{k \rightarrow \infty} E\left[f\left(\tilde{\mathbf{W}}_{k}\right)\right] \leq f^{*}+\lim _{k \rightarrow \infty} \epsilon_{k}=f^{*}+\epsilon .
$$

The condition $\lim _{k \rightarrow \infty} \frac{\epsilon_{k}-\epsilon_{k+1}}{\mu_{k}}=0$ of Theorem 1 requires that the rate of change in $\epsilon_{k}$ goes to zero faster than the step-size value $\mu_{k}$, as $k \rightarrow \infty$. This condition is satisfied for example when $\epsilon_{k}$ is kept constant, i.e., $\epsilon_{k}=\epsilon>0$ for all $k$.

By the convexity of $f$, we also have $f\left(E\left[\tilde{\mathbf{W}}_{k}\right]\right) \leq$ $E\left[f\left(\tilde{\mathbf{W}}_{k}\right)\right]$. Thus, as a special consequence of Theorem 1, we obtain $\limsup _{k \rightarrow \infty} f\left(E\left[\tilde{\mathbf{W}}_{k}\right]\right)-f^{*} \leq \epsilon$, showing that the accumulation points of $\left\{E\left[\tilde{\mathbf{W}}_{k}\right]\right\}$ are $\epsilon$-suboptimal. If $\epsilon=0$, then every accumulation point of the expected throughput $E\left[\tilde{\mathbf{W}}_{k}\right]$ is optimal. In addition, if $f$ has a unique minimum $\mathbf{x}^{*}$ over $\overline{\mathcal{R}}$, then $E\left[\tilde{\mathbf{W}}_{k}\right]$ converges to the minimum $\mathrm{x}^{*}$.

\section{Conclusions}

For the problem of utility optimal throughput allocations at a single scheduling station, we showed that choosing $\epsilon$-optimal solutions at every scheduling instance results in a long-term solution that is $\epsilon$-optimal in terms of utility maximization over the steady-state rate region. While we showed this for the decreasing step-size case, in future work we aim to show this also for the constant step-size case.

\section{REFERENCES}

[1] R. Agrawal and V. Subramanian. Optimality of certain channel aware scheduling policies. In Proceedings of 40th Annual Allerton Conf. Comm., Control, Comput., pages 1532-1541, Monticello, IL, October 2002.

[2] R. Agrawal, V. Subramanian, and R. Berry. Joint scheduling and resource allocation in CDMA systems. In Proceedings WiOPT, Cambridge, UK, March 2004.

[3] M. Andrews, K. K. Kumaran, K. Ramanan, A. L. Stolyar, R. Vijayakumar, and P. Whiting. Scheduling in a queueing system with asynchronously varying service rates. Probability in Engineering and Informational Sciences, 18(2):191-217, 2004.

[4] D. P. Bertsekas. Nonlinear Programming. Athena Scientific, Belmont, Mass., 1999.

[5] D. P. Bertsekas, A. Nedić, and A. Ozdalgar. Convex Analysis and Optimization. Athena Scientific, 2003.

[6] J. G. Dai and W. Lin. Maximum pressure policies in stochastic processing networks. Operations Research, 53(2):197-218, 2005.

[7] A. Eryilmaz and R. Srikant. Fair resource allocation in wireless networks using queue-length-based scheduling and congestion control. IEEE/ACM Transactions on Networking, 15:1333-1344, December 2007.

[8] R. G. Gallager. Discrete Stochastic Processes. Kluwer Academic Publishers, Boston, MA, 1995.

[9] L. Georgiadis, M. J. Neely, and L. Tassiulas. Resource allocation and cross-layer control in wireless networks. Foundations and Trends in Networking, 1(1):1-144, 2006.

[10] J. Huang, V. G. Subramanian, R. Agrawal, and R. Berry. Joint scheduling and resource allocation in downlink of OFDM systems. IEEE Trans. Wireless Comm. To appear.

[11] J. Huang, V. G. Subramanian, R. Agrawal, and R. Berry. Joint scheduling and resource allocation in uplink OFDM systems for broadband wireless access networks. IEEE JSAC, Special Issue on Broadband Access Networks. To appear.

[12] H. Kushner and P. Whiting. Asymptotic properties of proportional fair sharing algorithms. In Proceedings of 40th Annual Allerton Conf. Comm., Control, Comput., pages 1051-1059, Monticello, IL, October 2002.

[13] M. J. Neely. Dynamic Power Allocation and Routing for Satellite and Wireless Networks with Time Varying Channels. PhD thesis, Massachusetts Institute of Technology, 2003.

[14] E. Parzen. Stochastic Processes. Society for Industrial and Applied Mathematics. Holden-Day, Philadelphia, PA, 1999.

[15] B. T. Polyak. Introduction to optimization. Optimization Software Inc., 1987.

[16] R. T. Rockafellar and R. J.-B. Wets. Variational analysis, volume 317 of Grundlehren der Mathematischen Wissenschaften [Fundamental Principles of Mathematical Sciences]. SpringerVerlag, Berlin, 1998.

[17] A. L. Stolyar. Maximizing queueing network utility subject to stability: Greedy primal-dual algorithm. Queueing Systems, 50:401-457, 2005.

[18] A. L. Stolyar. On the asymptotic optimality of the gradient scheduling algorithm for multiuser throughput allocation. Oper. Res., 53(1):12-25, 2005.

[19] V. G. Subramanian. QoS and scheduling in wireless networks. Tutorial, IEEE VTC, Fall 2005, Dallas, Sept. 2005.

[20] L. Tassiulas and A. Ephremides. Stability properties of constrained queueing systems and scheduling policies for maximum throughput in multihop radio networks. IEEE Transactions on Automatic Control, 37:1936-1948, 1992. 